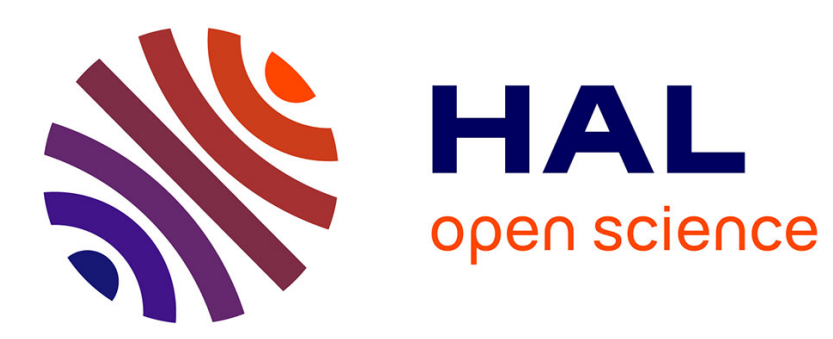

\title{
Front propagation in a vortex lattice: dependence on boundary conditions and vortex depth.
}

\author{
Edouard Beauvier, Simona Bodea, Alain Pocheau
}

\section{To cite this version:}

Edouard Beauvier, Simona Bodea, Alain Pocheau. Front propagation in a vortex lattice: dependence on boundary conditions and vortex depth.. Soft Matter, 2016, 12, pp.8935-8941. 10.1039/c6sm01547f . hal-01406078

\section{HAL Id: hal-01406078 \\ https://hal.science/hal-01406078}

Submitted on 22 May 2018

HAL is a multi-disciplinary open access archive for the deposit and dissemination of scientific research documents, whether they are published or not. The documents may come from teaching and research institutions in France or abroad, or from public or private research centers.
L'archive ouverte pluridisciplinaire HAL, est destinée au dépôt et à la diffusion de documents scientifiques de niveau recherche, publiés ou non, émanant des établissements d'enseignement et de recherche français ou étrangers, des laboratoires publics ou privés. 


\title{
Front propagation in a vortex lattice : dependence on boundary con- ditions and vortex depth ${ }^{\dagger}$
}

\author{
E. Beauvier, ${ }^{a}$ S. Bodea, ${ }^{a}$ and A. Pocheau ${ }^{a}$
}

\author{
Received Xth $X X X X X X X X X X 20 X X$, Accepted Xth $X X X X X X X X X 20 X X$ \\ First published on the web Xth $X X X X X X X X X X 200 X$ \\ DOI: 10.1039/b000000x
}

\begin{abstract}
We experimentally address the propagation of reaction-diffusion fronts in vortex lattices by combining, in a Hele-Shaw cell and at low Reynolds number, forced electroconvective flows and an autocatalytic reaction in solution. We consider both vortex chains and vortex arrays, the formers referring to mixed free/rigid boundary conditions for vortices and the latters to free boundary conditions. Varying the depth of the fluid layer, we observe no variation of the mean front velocities for vortex arrays and a noticeable variation for vortex chains. This questions the two-dimensional character of front propagation in low Reynolds number vortex lattices, as well as the mechanisms of this dependence.
\end{abstract}

\section{Introduction}

Stirred flows are well-known to enhance the transport properties of a medium by their ability in intimately mixing components. This feature extends to reacting flows, as the net reaction time of a medium is found to sharply decrease with the stirring rate. In particular, in regimes where reaction proceeds by the propagation of fronts separating reacted and unreacted zones, the mean front velocity is found to largely increase with the vortex intensity. Important consequences follow in the fields of combustion ${ }^{1}$, chemistry ${ }^{2,3}$ or even ecology ${ }^{4-6}$. However, the actual mechanisms of front velocity enhancement as well as the whole factors on which this velocity depends call for a better understanding in both turbulent ${ }^{1,7-12}$ and laminar ${ }^{13-21}$ contexts.

We focus attention here on laminar single scale flows made of a periodic planar array of vortices. Propagation of fronts in this context has been addressed by theories ${ }^{13-16}$ and numerical simulations ${ }^{14-17}$ by considering a two-dimensional array of vortices involving equal width and length. The same boundary conditions (hereafter called b.c.) were thus displayed on all their separatrices (actually the free b.c. provided by neighbor vortices) and the set of separatrices was found to form a square array. In comparison, apart from a study in Taylor vortex flows ${ }^{21}$, experiments ${ }^{18-20}$ addressed a single chain of vortices confined in a channel. Then, different boundary conditions were applied on vortex separatrices depending on whether they were a channel wall (rigid b.c.) or a frontier with

$\dagger$ Electronic Supplementary Information (ESI) available: Movies of front propagation in a channel accelerated 25 times. Movie "FreeBC" : large channel (5 vortices between walls) and free effective boundary conditions, $U / V_{0}=35$. Movie "RigidBC" : narrow channel (1 vortex between walls) and rigid effective boundary conditions, $U / V_{0}=27$. See DOI: $10.1039 / \mathrm{b} 000000 \mathrm{x} /$ ${ }^{a}$ Aix Marseille Univ, CNRS, Centrale Marseille, IRPHE, Marseille, France a neighbor vortex (free b.c.). In addition, all experiments naturally took place in a fluid layer whose depth $d$ was thus a natural parameter of them.

Usually, experiments involved a single vortex depth $d$. However, one of them varied this parameter and concluded that the mean front velocity $V_{f}$ not only depends on the vortex intensity but also on the vortex depth $d^{19,20}$. As it was performed in a vortex chain, it involved mixed free/rigid boundary conditions on vortices. Its vortices were also longer than large, so that the set of separatrices was rectangular instead of square ${ }^{19,20}$. Accordingly, this experiment involved two kinds of asymmetry : (i) a boundary asymmetry yielding different b.c. on a vortex, (ii) a geometric asymmetry induced by the difference between vortex width and vortex length.

As theories or simulations considered a two-dimensional planar flow, they implicitly assumed a 2-dimensional character of front propagation in a vortex lattice ${ }^{13-17}$. However, the dependence of $V_{f}$ on $d$ questions this assumption and raises the following interrogations regarding its possible origin : could it follow from the geometric asymmetry of vortices or from their boundary asymmetry and then, by what kind of mechanism ? In particular, could it refer to a modification of the structure of a still planar flow or to the occurrence of secondary flows breaking the planar flow assumption?

To answer these questions, we perform here front propagation experiments in an array of vortices involving equal width and length and either similar or different boundary conditions on their separatrices. We then vary the channel depth so as to highlight the implications of geometrical symmetry and of boundary symmetry on the dependence of front propagation on channel depth. Altogether, this provides the first comparison of the effects of boundary conditions and of vortex depths on front propagation in a stirred medium. 
The experimental set-up allowing these configurations to be achieved is reported in section 2 . We then address qualitative and quantitative results on section 3 and discuss them in section 4. A conclusion about the study and its general issue is drawn in section 5 .

\section{Experiment}

The experiment is conducted in a fluid enclosed in a channel with top, bottom and lateral boundaries and whose depth $d$ is imposed by spacers (Fig.1-a). Three depths have been considered : $d=2,4$ or $6 \mathrm{~mm}$.

Flows are induced by electroconvection thanks to both a pattern of magnets placed below the channel and an electric current imposed by electrodes placed at the extremities of the fluid layer (Fig.1-a). All the magnets are squares with dimension $L=20 \mathrm{~mm}$. They are placed one beside the others so as to form a square lattice. Their magnetization direction alternates between neighbors. It thus induces in the fluid layer a periodic magnetic field and thus periodic Laplace forces which give rise to alternating vortices. Electromagnetic forcing has been used for a while in many different contexts ranging from boundary layer effects in salty water ${ }^{22,23}$, mixing ${ }^{24,25}$, single scale flows ${ }^{26-28}$ multi-scale flows ${ }^{29}$ or quasi-two dimensional turbulent or chaotic flows ${ }^{30-32}$. Beyond hydrodynamics, it has also been used to study front propagation ${ }^{18-20}$ in a laminar regime. In particular, in the Stokes regime in which our experiment fits, the current density, the magnitude of the Laplace force density and then the vortex intensity are all proportional, thereby providing a simple mean to tune the flow magnitude by the current intensity.

Front propagation is provided by an autocatalytic oxydoreduction reaction between chlorite and iodure ions in the fluid layer :

$$
\begin{aligned}
& \mathrm{ClO}_{2}^{-}+4 \mathrm{I}^{-}+4 \mathrm{H}^{+} \rightarrow \mathrm{Cl}^{-}+2 \mathrm{I}_{2}+2 \mathrm{H}_{2} \mathrm{O} \\
& 5 \mathrm{ClO}_{2}^{-}+2 \mathrm{I}_{2}+2 \mathrm{H}_{2} \mathrm{O} \rightarrow 5 \mathrm{Cl}^{-}+4 \mathrm{IO}_{3}^{-}+4 \mathrm{H}^{+} \\
& \mathrm{IO}_{3}^{-}+5 \mathrm{I}^{-}+6 \mathrm{H}^{+} \rightarrow 3 \mathrm{I}_{2}+3 \mathrm{H}_{2} \mathrm{O}
\end{aligned}
$$

The autocatalytic feature is provided by the fact that the consumption of iodide $I^{-}$worked out in (1) is enhanced in (3) by a product of reaction, the iodate $\mathrm{IO}_{3}^{-}$produced in (2) thanks to the iodine $I_{2}$ provided by the consumption of iodide itself in (1) and (3). This provides a positive feed-back of iodide consumption on itself possibly yielding a run-away and, in specific regimes, a reaction zone localized in a thin interface, the front, standing in between reacted and unreacted zones ${ }^{33,34}$. The detection of these zones, and thus of the front as their frontier, is provided by a change of colour as reaction proceeds. For this, a Starch indicator introduced in the solution forms a blue complex with iodide and iodine which disappears after reaction following the depletion of iodide. The unreacted blue zone then transforms into an uncolored reacted zone. The front velocity may thus be deduced from the kinematics of their frontier, as recorded on a computer by a camera (Fig.2). In a fluid at rest, this velocity is labelled $V_{0}$ and amounts in our experiment from 1.0 to $1.4 \mathrm{~mm} / \mathrm{mn}$ depending on the imposed current density ${ }^{19,20}$. For this, the temperature has been stabilized to $20^{\circ} \mathrm{C}$ to minimize the variability of $V_{0}$ to less than few percents.

When the medium is stirred by the vortex lattice, front propagation may take advantage of advection. This is in particular so when the front is enrolled by vortices. Then, following the large value of the flow velocity compared to the proper front velocity $V_{0}$ (from 5 to 40 times greater here), the front velocity stays close to it. In particular, it appears that at large flow velocity, after having crossed a vortex separatrix, the front is advected on a streamline (Fig.2) where its mean velocity provides a direct information on the vortex intensity $U$. Thanks to the linear relationship between flow velocity and current density, this determination may then be extended on the whole range of the experiment.

Considering a planar vortex flow $\mathbf{V}$, its advective term V. $\nabla \mathbf{V}$ may be estimated to about $U^{2} / L$ in the bulk since the variation scale in the flow plane is then $L$. However, close to a lateral boundary, this scale decreases to about the channel depth $d$, yielding another estimate of about $U^{2} / d$ there. In both cases, the dissipative term $v \triangle \mathbf{V}$ may be estimated to $v U / d^{2}$ for a near Poiseuille profile where $v \approx 1 \mathrm{~mm}^{2} / \mathrm{s} \mathrm{de}$ notes the water viscosity. Accordingly, the Reynolds number reads $R e=U^{2} d^{2} / v U d \equiv U d / v$ close to a boundary and $R e^{\prime}=\operatorname{Re} d / L<R e$ in the bulk. As $L=20 \mathrm{~mm}$, one gets in our experimental range $U \leq 40 \mathrm{~mm} / \mathrm{mn}, 2 \leq d \leq 6$, a maximal Reynolds number $R e^{\prime}=1.2$ in the bulk and $R e=4$ close to boundaries. Accordingly, the experiment may be considered to stand within or close to the Stokes regime.

The channel is $400 \mathrm{~mm}$ long and either 20,80 or $100 \mathrm{~mm}$ large. In particular, its width could then include 1, 4 or 5 magnets. Placing the channel sides on magnet boundaries, this provided 1, 4 or 5 vortices in between them (Fig.1-b,c). As these vortices display equal length and width, they satisfy the geometrical symmetry. When a single vortex fits in between the channel width, it undergoes rigid b.c. on the separatrices made by the channel sides and free b.c. on the remaining ones made by the frontier with neighbor vortices. This mixed case corresponds to asymmetric boundaries (Fig.1-b). In contrast, when the channel width is large compared to the vortex size, the experiment shows that front propagation in the central vortices is favored, so that they act as a leading zone for the overall propagation (Fig.2-a). As these central vortices are surrounded by other vortices, their b.c. are free on all their separatrices. This corresponds to symmetric boundaries (Fig.1-c).

This way, the set-up is able to provide an experimental achievement of front propagation with geometric symmetry 


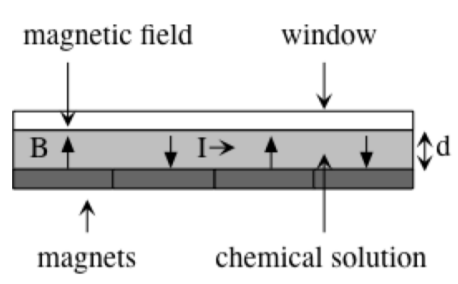

(a)

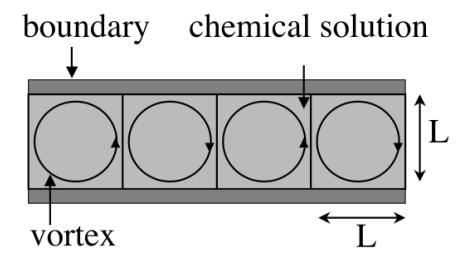

(b)

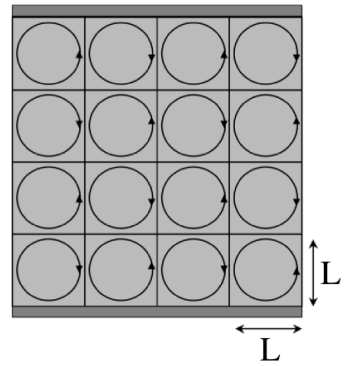

(c)

Fig. 1 Sketch of the set-up showing the chemical solution stirred by Laplace forces induced by an electric current flowing over alternate magnets (a) and the resulting chain (b) or array (c) of counter-rotating vortices. The magnetic field and the electric current are labelled $\mathbf{B}$ and $I$ and the channel depth and the vortex size $d$ and $L$ respectively. Vortex boundary conditions are mixed free/rigid (b) or free (c), depending on whether vortices make contact with the channel sides (b) or are surrounded by other vortices (c; except for side vortices).

and either symmetric or asymmetric boundaries.

\section{Fronts and Velocity}

We report typical evolutions of fronts in the vortex lattice together with quantitative measurements of their mean front velocity $V_{f}$ along the channel axis, at various vortex intensity $U$ and channel depths $d$. The two situations of free b.c. (symmetric vortices) and mixed free/rigid b.c. (asymmetric vortices), are considered to allow for their comparison.

\subsection{Symmetric vortices : free b.c.}

Here, the channel width englobes 4 or 5 vortices, so that 2 or 3 central vortices undergo free b.c. on all their separatrices (Fig.1-c). A typical evolution of reaction fronts is reported on figure 2-a. It appears that the fronts display a periodic motion involving alternating phases between a slow crossing of separatrices and a quick advection in between. In addition, the contamination of a new line of vortices always occur on central vortices so that the mean front propagation is actually set by symmetric vortices.

Figure 3-a reports, at various channel depths, the enhancement of the mean front velocity $V_{f}$ by the flow in term of nondimensional variables $V_{f} / V_{0}, U / V_{0}$. It appears no definite dependence on the channel depth $d$, the velocity enhancement displaying the same linear trend in this velocity range. This denies relevance of the channel depth $d$ on the mean front propagation, in agreement with a two-dimensional view of this propagation issue.

\subsection{Asymmetric vortices : mixed free/rigid b.c.}

Here, the channel width englobes only a single vortex which thus undergoes mixed free/rigid b.c (Fig.1-b). Regarding front motion, the typical evolution of reaction fronts reported on figure 2-b shows that the quick advection phase occurs near the channel sides. As in the previous symmetric situation, fronts undergo a periodic motion made of a succession of slow crossing of separatrices and quick advection in between, but the latter occurs close to a rigid b.c. this time.

Figure 3-b reports, at various channel depths, the enhancement of the mean front velocity $V_{f}$ by the flow in term of non-dimensional variables $V_{f} / V_{0}, U / V_{0}$. It shows evidence of a dependence on the channel depth $d$, the velocity enhancement displaying an increasing concavity with the channel depth. This reduction of velocity enhancement at large $d$ yields in particular, at $U / V_{0} \approx 26$, a decrease of about $40 \%$ of the mean front velocity $V_{f}$ when increasing the channel depth from $d=2 \mathrm{~mm}\left(V_{f} / V_{0}=11.7\right)$ to $d=6 \mathrm{~mm}\left(V_{f} / V_{0}=6.8\right)$. This evidences a noticeable influence of the channel depth on front propagation, a fact which questions the two-dimensional assumption considered in theories and simulations ${ }^{13-17}$.

\section{Discussion}

Experimental data on velocity enhancement by vortices reveal an ambivalent behavior regarding the dependence on the channel depth : no dependence for free b.c. ; a noticeable dependence for mixed free/rigid b.c.. As these data have been obtained on vortices involving equal length and width, this indicates that their dependence on $d$ has to be traced back not to geometrical asymmetry ${ }^{19,20}$ but to the asymmetry between the boundary conditions applied at the vortex periphery.

For all vortices, rigid b.c. are in order at the top and bottom plates. However, for mixed free/rigid b.c., rigid b.c. are also undergone at two opposite separatrices of vortices. We discuss the implications of these features on front propagation and especially its evolution with the channel depth. 


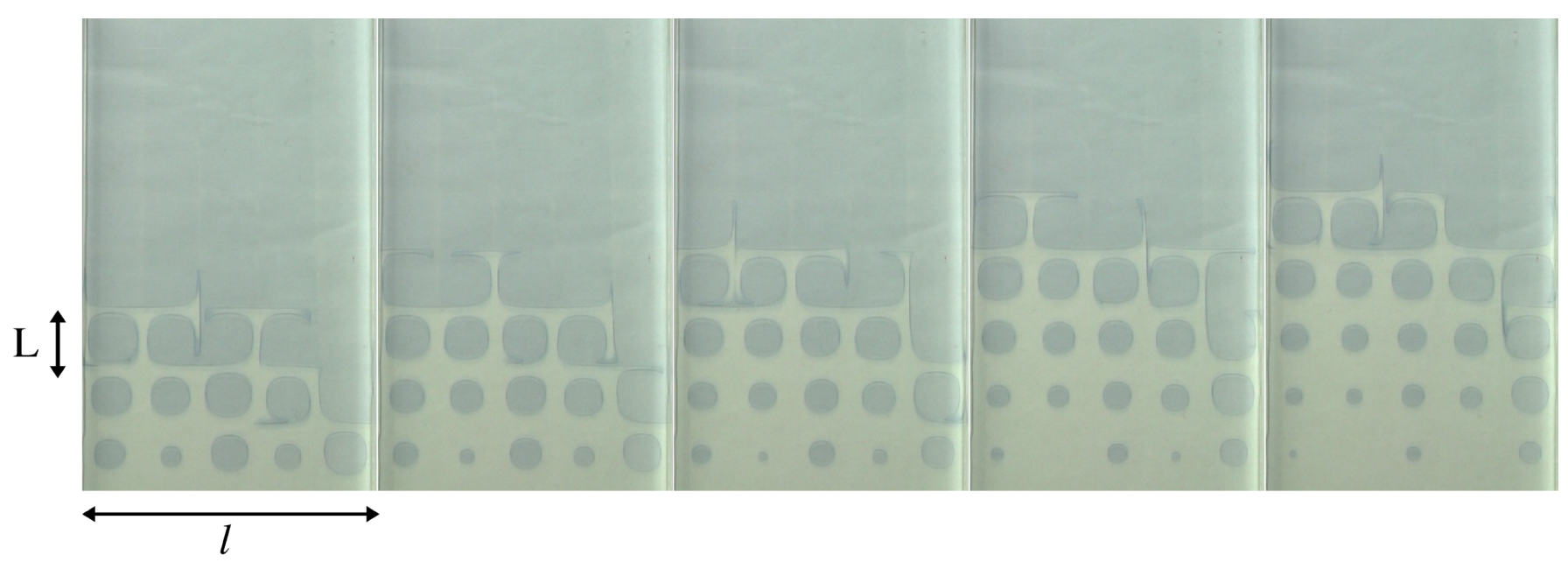

(a)
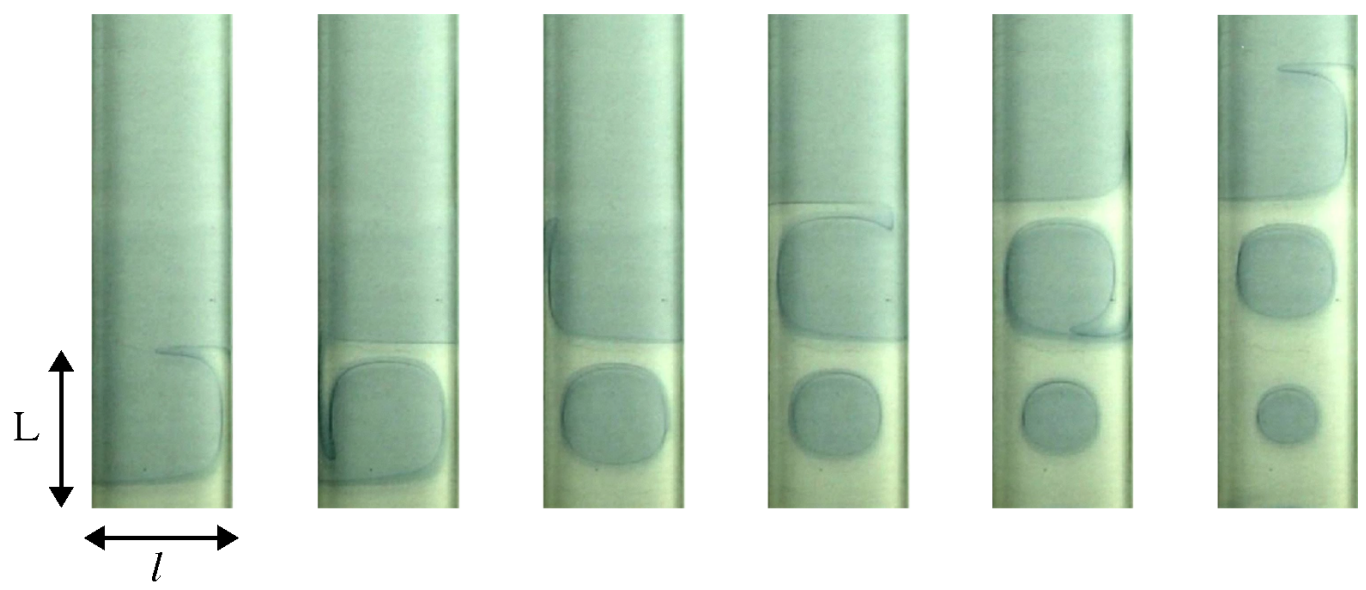

(b)

Fig. 2 Front propagation in a vortex array (a) or in a vortex chain (b). Times regularly increases from left to right while fronts propagate upwards. The vortex size is $L=20 \mathrm{~mm}$, the channel depth is $d=2 \mathrm{~mm}$ and the channel width is labelled $l$. (a) Free boundary conditions, except for the side vortices. $l / L=5, U / V_{0}=17.3$. Time interval between pictures : 65 s. (b) Rigid lateral boundary conditions. $l / L=1$, $U / V_{0}=14.6$. Time interval between pictures : 58 s.

\subsection{Symmetric vortices : free b.c.}

The rigid b.c. on the top and bottom plates of the channel imply a near parabolic profile of the flow field on the channel depth direction $\mathbf{z}$. Nevertheless, front propagation shows no dependence on its extent $d$. This feature is coherent with :

1. planar flows involving a common structure $\mathbf{U}$ independent of $z$ and $d$ and an intensity simply varying with $z$ on the depth axis : $\mathbf{V}=f(z / d) \mathbf{U}$, the flow $\mathbf{U}$ belonging to the plane $(x, y)$ and being only dependent on its variables $: \mathbf{U} \equiv \mathbf{U}(x, y)$.

2. a front propagation monitored by the planar flow $\mathbf{U}$, independently of the z-profile $f(z / d)$.

The first feature is reminiscent of a Poiseuille flow and may be thought to be satisfied for electroconvective flows at the dominant order. The second feature follows from the local relationship between front velocity and flow, as studied on a 


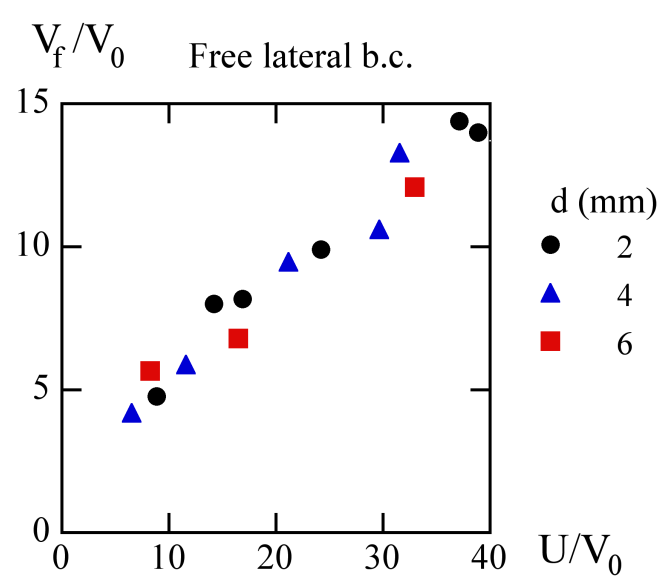

(a)

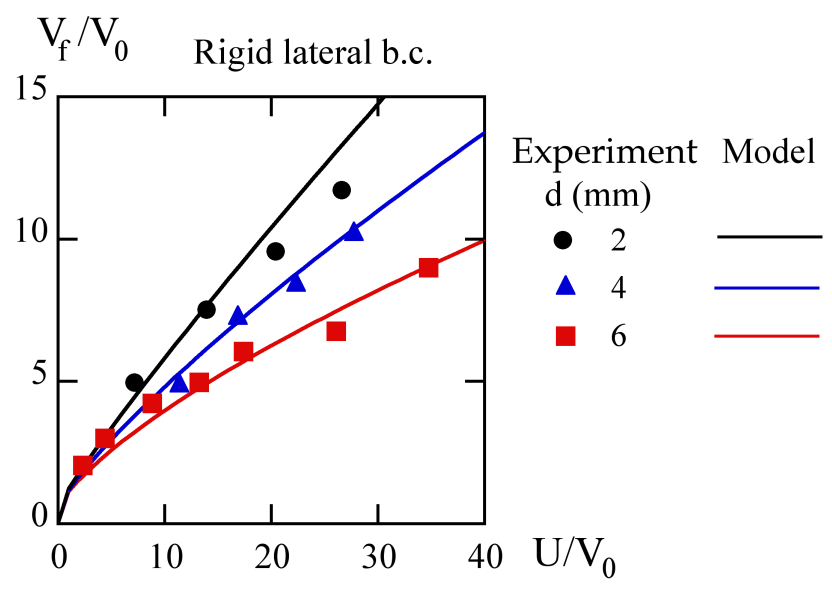

(b)

Fig. 3 Reduced front velocity $V_{f} / V_{0}$ as a function of the reduced vortex intensity $U / V_{0}$. (a) Free b.c. : a single linear relationship is displayed whatever the channel depth $d$. (b) Mixed free/rigid b.c. : concave relationships are displayed with a concavity increasing with the channel depth $d$. Lines correspond to predictions from an optimization model ${ }^{19,20}$.

Poiseuille flow ${ }^{35,36}$. It was then found that front velocity turns out to be the sum of the proper front velocity $V_{0}$ plus either the mean flow velocity on the channel depth for low enough $d$ compared to the front thickness (i.e. $\eta=d V_{0} / 2 D<1$ ) or the maximal flow velocity in the opposite case ${ }^{35,36}, D=$ $2.10^{-3} \mathrm{~mm}^{2} / \mathrm{s}$ denoting the molecular diffusivity of the relevant species. In all cases, front velocity appears as the sum of $V_{0}$ and a velocity linearly related to $U$ by a constant prefactor. In particular, in our experiment, as $\eta$ is larger than 8 , fronts locally advance at velocity $V_{0}+\mathbf{U}(x, y)$.n where $\mathbf{n}$ denotes the front normal in the mid-depth plane $z=d / 2$ and where the $\mathrm{Z}$-profile has been normalized so that $f(1 / 2)=1$.

Altogether, both features may thus be thought as relevant for explaining the independence of mean front velocity on $d$.

\subsection{Asymmetric vortices : mixed free and rigid b.c.}

In comparison to the above case of symmetric vortices, the dependence of mean front velocity on $d$ displayed when some vortex boundaries are rigid calls for either a structure of planar flows dependent on $d$ or a breaking of the planar flows assumption.

\subsubsection{Dependence of planar flows on $d$.}

This issue has been explored in a previous study where the mean front velocity was argued to correspond to the quickest trajectory of the front head in the vortex field ${ }^{19,20}$. Then, an optimization model based on a simplified family of trajectories inspired by observations was worked out to derive the mean front velocity within a prescribed modeling of planar flows. In particular, these flows involved circular streamlines and boundary layer thicknesses of half the channel depth. The latter feature was motivated by the fact that, in a Hele-Shaw cell, the channel depth monitors the width of boundary layers, following the dominant dissipation implied by the presence of parallel plates. Its value was then taken to be $d / 2$, yielding the quickest streamline to be located at this distance to the channel wall. This constraint thus led the flow field to depend on $d$. Its implication on the mean front velocity was deduced by selecting the trajectory that minimizes the crossing time through a vortex.

The predictions of the optimization model for geometrically symmetric vortices are reported on figure 3-b for the three channel depths. They are found to satisfactorily recover the experimental data, especially the increasing concavity of the enhancement curves with the channel depth. Beyond this agreement, it should be realized that the optimization model is based on a phenomenological family of front trajectories and on a crude flow modeling. In particular, the circular form of streamlines stands for an approximation of actual elliptical streamlines, which prevents the flow velocity to vary on them. In this sense, the streamline velocity is intended to reflect the average velocity on it instead of its local values. It thus corresponds to the nature of the velocity measured experimentally and is sufficient to evaluate the advection time of the front head on a streamline. However, this approximation also implies circular streamlines which are obviously inadequate around the square vortex separatrices, especially around the vortex corners. Finally, the scaling of the boundary layer thickness with the channel depth, although physically sound, 
involves an approximate prefactor.

For these reasons, the optimization model must be taken as a toy model whose merit is to provide a kinematic origin for the dependence of front velocity on the channel depth : as the quickest front trajectory proves to belong to the boundary layer ${ }^{19,20}$, front propagation appears to be sensitive to its structure and thus to the channel depth which modifies its thickness. However, beyond this qualitative relevance, the optimization model appears too crude for allowing a quantitative comparison. In particular, as its major outcome is the sensitivity of front propagation to the structure of the boundary layer, it actually calls for an accurate flow modeling to provide a relevant quantitative comparison. In addition, as it relies on a restricted family of trajectories, its predicted mean flow velocities would nevertheless be approximate. Accordingly, given an accurate model of planar flow, a relevant quantitative comparison to experiment would call either for an extension of the optimization model to any possible trajectory or for numerical simulations.

\subsubsection{Breaking of the planar flows assumption.}

For rotating flows, a generic mechanism for the generation of secondary flows is provided by the Ekman pumping ${ }^{37}$. It relies on the fact that viscous effects at boundaries make velocity and centrifugal force shrink whereas pressure is maintained. This therefore induces a force disequilibrium in the fluid which results in setting the fluid into motion. Another possible origin for secondary flows is the occurrence of hydrodynamical instabilities but these are not expected at the low Reynolds numbers at which the experiment stands $(R e=U d / v<4$ here ; see section 2). An important difference between these two mechanisms should be noticed: whereas the Ekman pumping must be present at all Reynolds numbers or at none, instabilities manifest themselves above some onset. Accordingly, if secondary flows are generated by Ekman pumping, their amplitude should continuously grow with the vortex intensity without onset.

In our configuration, this phenomenon underlying the Ekman pumping might first occur at the top and bottom rigid plates of the channel. It should thus induce inward flows towards the vortex centers giving rise, by incompressibility, to flows directed normally to the plates and finally to two spiral flows, following the top-bottom symmetry of the configuration. These secondary flows should however be equally present on symmetric and asymmetric vortices, i.e. for free or mixed free/rigid b.c., since both configurations involve top and bottom plates. No spirals have however been detected from front motion in our experimental range so that the corresponding secondary flows are presumably too faint to noticeably modify front dynamics. Anyway, they could not solely explain the specificity of the asymmetric vortex configuration, i.e. its noticeable dependence on the channel depth.

In contrast, the presence of rigid lateral b.c. stands as a specificity of asymmetric vortices from which specific inward flows could be generated. They might in particular modify the advection of the front head all along its travel inside the lateral boundary layer and possibly slightly deflect its trajectory towards the vortex center. This could result, at the end of the advection phase, in a slight increase of the front distance to the neighbor vortex separatrix. As the front must cross it by its sole propagation at its slow proper velocity $V_{0}$, this effect, although faint, could result in a noticeable decrease of the mean front velocity. This mechanism is however in competition with the one induced by the increase of the boundary layer thickness of planar flows, since both yield an increase with $d$ of the distance of the front head to the neighbor vortex separatrix. Determining the role of each thus calls for a quantitative evaluation of their respective effects.

Beyond the qualitative explanation of secondary flow generation, it should be noticed that a quantitative determination of their amplitude requires first an accurate modeling of the primary flows, i.e. of planar flows. Accordingly, the discrimination between the two above mechanisms calls for an accurate modeling of planar electroconvective flows in both symmetric (free b.c.) and asymmetric vortices (mixed free/rigid b.c.). Then, the most direct way to determine the implications of these flows on front propagation will be to perform numerical simulations. Comparison with experiments could then be able to conclude whether the planar flows for free b.c. as well as their modification by lateral walls allow to recover both the independence to the channel depth $d$ for symmetric vortices as well as the dependence on it for asymmetric vortices.

\section{Conclusion}

In theories and simulations, front propagation in laminar flows is addressed on two-dimensional flows involving a prescribed structure $^{13-17}$. This assumption actually denies a relevant influence of three-dimensional features of vortices as their depth or asymmetries induced by boundary conditions or by direct forcing. However, a previous experiment performed on a vortex chain enclosed in a channel revealed a variation of the mean front velocity with the channel depth ${ }^{19,20}$. This observation thus calls for determining to what extent front propagation in vortex arrays may be considered as 2-dimensional and to what kind of features it may be dependent.

To address this issue, we performed experiments so as to draw the first comparison of front propagation for different flow b.c. : the free b.c. considered in theories or simulations and the mixed free/rigid b.c. involved in the experiment which evidenced a dependence on the channel depth. For this, we considered both narrow and large channels as compared to the 
vortex width. Large channels provided effective free b.c., as they leave place for several vortices between their walls (vortex array). Narrow channels provided mixed free/rigid b.c. for vortices, as they englobe a single line of vortices between their walls (vortex chain). In both cases and in contrast with a previous experiment ${ }^{19,20}$, we considered only geometrically symmetric vortices involving equal width and length, as considered in theories and simulations.

The dependence of mean front velocity on channel depth $d$ has been found to be bivalent : no dependence for vortex arrays (free b.c.) but a noticeable dependence for vortex chains (mixed free/rigid b.c.). In particular, in the latter case, the mean front velocity was found to increase as $d$ was reduced, up to eventually reach at low $d$ the values found on vortex arrays. Thus, in contrast with primary intuition, the most dissipative configuration regarding hydrodynamics (vortex chain at low cell depth) provided a similar efficiency regarding propagative transport as the less dissipative one (vortex array). This might provide a mean for restoring in confined systems a propagation efficiency similar to that displayed in unconfined ones. In particular, this property might apply to chemical engineering in stirred systems, possibly even down to microfluidic scales ${ }^{38}$. It would then stress that the net reaction efficiency in narrow geometries could be monitored by their depth, up to eventually recover in thin configurations the efficiency involved in extended ones.

On the other hand, on vortex arrays, the independence of front velocity from the channel depth shows that the vertical profile of flows plays no significant role regarding propagation efficiency. This property might apply to natural situations which proceed by reaction-diffusion-advection within a set of quasi two-dimensional vortices, such as in plankton dynamics ${ }^{4,5}$ or in ozone hole evolution ${ }^{6}$. It would then point out the planar structure of their vortices as the essential feature regarding the global dynamics.

Finally, the analysis of the possible origin of the dependence of front propagation on channel depth in vortex chains (mixed free/rigid b.c.) has stressed two possible mechanisms : a modification of planar flows by boundary conditions or a breaking of the planar flow assumption by secondary flows. In the first case, front propagation would still be considered as a two-dimensional issue, sensitive enough for being influenced by b.c. ; in the latter case, it could no longer be considered as two-dimensional since noticeably dependent on faint threedimensional features. Discrimination between these mechanisms calls for an accurate modeling of electroconvective planar flows together with simulations of front propagation, so as to provide relevant comparisons to experiments.

\section{Acknowledgments}

This work has been carried out in the framework of the Labex MEC (ANR-10-LABX-0092) and of the A*MIDEX project (ANR-11-IDEX-0001-02), funded by the Investissements d'Avenir French Government program managed by the French National Research Agency (ANR).

\section{References}

1 F. A. Williams, Combustion Theory, Benjamin-Cummings, New York, 1985.

2 R.J.Field and M. Editors, Oscillations and travelling wave in chemical systems, John Wiley and Sons, New-York, 1985.

3 I. R. Epstein, Nature (London), 1995, 374, 231.

4 E. R. Abraham, Nature (London), 1998, 391, 577.

5 E. R. Abraham, C. S. Law, P. W. Boyd, S. J. Lavender, M. T. Maldonado and A. R. Bowie, Nature, 2000, 407, 727.

6 S. Edouard, B. Legras, F. Lefèvre and R. Eymard, Nature (London), 1996, 384, 444.

7 G. I. Sivashinsky, Comb. Sci. tech., 1988, 62, 77.

8 V. Yakhot, Comb. Sci. tech., 1988, 60, 191.

9 A. Pocheau, Phys. Rev. E, 1994, 49, 1109.

10 P. D. Ronney, B. D. Haslam and N. O. Rhys, Phys. Rev. Lett., 1995, 74, 3804.

11 A. Pocheau and D. Queiros-Condé, Phys. Rev. E, 1996, 76, 3352.

12 S. S. Shy, R. H. Jang and P. D. Ronney, Combust. Sci. Tech., 1996, 113, 329-350.

13 B. Audoly, H. Berestycki and Y. Pomeau, C. R. Acad. Sci, Sériellb, Paris, 2000, 328, 255.

14 M. Abel, A. Celani, D. Vergni and A. Vulpiani, Phys. Rev. E, 2001, 64, 0466307.

15 M. Abel, M. Cencini, D. Vergni and A. Vulpiani, Chaos, 2002, 12, 481-488.

16 M. Cencini, A. Torcini, D. Vergni and A. Vulpiani, Phys. Fluids, 2003, 15, 679-688.

17 N. Vladimirova, P. Constantin, A. Kiselev, O. Ruchayskiy and L. Ryzhik, Combustion Theory Modelling, 2003, 7, 485-508.

18 M. S. Paoletti and T. H. Solomon, Phys. Rev. E, 2005, 72, 046204-046213.

19 A. Pocheau and F. Harambat, Phys. Rev. E, 2006, 73, 065304-065306.

20 A. Pocheau and F. Harambat, Phys. Rev. E, 2008, 77, 036304-036332.

21 B. Thompson, J. Novak, M. Wilson, M. Britton and A. Taylor, Phys. Rev. E, 2010, 81, 047101-4. 
22 L. Rossi and J.-P. Thibault, Journal Of Turbulence, 2002, $3,1-15$.

23 J.-P. Thibault and L. Rossi, Journal of Physics D: Applied Physics, 20032, 36, 2559-2568.

24 O. Cardoso and P. Tabeling, Eur. J. Mech., B/Fluids, 1989, 8(6), 459-470.

25 O. Cardoso, B. Gluckmann and a. P. T. O. Parcollet, Phys. Fluids, 1996, 8, 209-214.

26 R. Akkermans, A. Cieslik, L. Kamp, R. Trieling, H. Clercx and G. J. F. van Heijst, Phys. Fluids, 2008, 116601-15.

27 M. Duran-Matut, R. R. Trieling and G. J. F. van Heijst, Phys. Rev. E, 2011, 83, 0163069.

28 D. Kelley and N. Ouellette, Phys. Fluids, 2011, 23, 054103-10.

29 L. Rossi, J. C. Vassilicos and Y. Hardalupas, J. Fluid Mech., 2006, 558, 207.

30 P. Tabeling, S. Burkhart, O. Cardoso and H. Willaime, Phys. Rev. Lett., 1991, 675, 3772-3775.

31 H. Clercx, G. J. F. van Heijst and M. L. Zoeteweij, Phys. Rev. E, 2003, 67, 066303-9.

32 N. T. Ouellette and J. P. Gollub, Phys. Rev. Lett., 2007, 99, 194502-4.

33 A. Saul and K. Showalter, Oscillations and travelling Waves in chemical system, Ed. Field and Burger, 1985, Chap.11, 419-439.

34 A. Hann, A. Saul and K. Showalter, J. Am. Chem. Soc., 1982, 104, 3838-3844.

35 B. F. Edwards, Phys. Rev. Lett., 2002, 89, 104501-104505.

36 M. Leconte, J. Martin, N. Rakotomalala and D. Salin, Phys. Rev. Lett., 2003, 90, 128302.

37 V. W. Ekman, Arch. Math. Astron. Phys., 1905, 2, 1-52.

38 A. de Mello, Nature, 2006, 442, 394-402.

8 | Journal Name, 2010, [vol],1-8 